\title{
EDITORIAL
}

\section{ACTIVIDAD FÍSICA, EJERCICIO FÍSICO Y CONDICIÓN FÍSICA EN EL ÁMBITO DE LA SALUD PÚBLICA}

\section{Yolanda Escalante}

Facultad de Ciencias del Deporte. Grupo de Investigación AFIDES. Universidad de Extremadura.

El cuerpo del ser humano está preparado para moverse, más aún, tiene la necesidad de hacerlo. No obstante, a pesar de esta necesidad, la práctica de actividad física en la población es cada vez menos frecuente, muy especialmente en niños ${ }^{1}$. Estos bajos niveles de práctica de actividad física conllevan el sedentarismo de buena parte de la población, lo que unido a unos hábitos alimentarios nada idóneos han provocado el aumento alarmante de diferentes patologías (obesidad, enfermedades cardiovasculares, síndrome metabólico...) que, en algunos casos, se han convertido en un auténtico problema de Salud Pública. Uno de los trabajos que se publican en este número ${ }^{2}$ concluye que existen valores elevados de prevalencia de la hipertensión en la población adulta de la Comunidad Madrid (29\% en total, $35 \%$ en hombres y $24 \%$ en mujeres). Para luchar contra el aumento de la prevalencia de estas patologías, Instituciones Internacionales, Gobiernos y Comunidades Autónomas han articulado diferentes estrategias. Una de las más recurrentes es

\footnotetext{
Correspondencia

Yolanda Escalante

Facultad de Ciencias del Deporte. Grupo de Investigación AFIDES

Universidad de Extremadura.

Avda. Universidad s/n. 10071 - Cáceres. España

Correo electrónico: yescgon@unex.es
}

apoyar, promocionar y perseguir el aumento de la práctica de la actividad física ${ }^{3}$. En su definición clásica, la actividad física es "cualquier movimiento corporal producido por los músculos esqueléticos que produzca un gasto energético mayor al existente en reposo" ". Con esta definición en mente, se puede constatar que las recomendaciones de práctica de actividad física semanal han ido variando en las últimas décadas, hasta llegar $\mathrm{a}^{3}$ :

- Los niños de entre 5 y 17 años deberían acumular un mínimo de 60 minutos diarios de actividad física, mayoritariamente aeróbica, de intensidad moderada o vigorosa. Asimismo, sería conveniente un mínimo de tres veces semanales de práctica de actividades que fortalezcan el aparato locomotor.

- Las personas adultas de entre 18 y 64 años deberían acumular un mínimo de 150 minutos semanales de actividad física aeróbica moderada, o bien 75 minutos semanales de actividad física aeróbica vigorosa (o la combinación equivalente de ambas). Para obtener mayores beneficios sobre la salud se debería llegar a 300 y 150 minutos de actividad aeróbica moderada o vigorosa respectivamente. Asimismo, sería conveniente un mínimo de dos o tres veces 
semanales de práctica de actividades que fortalezcan el aparato locomotor.

- Las personas adultas mayores de 65 años deberían añadir tres veces semana de práctica de actividades para mejorar el equilibrio y prevenir caídas a las recomendaciones indicadas a los adultos de entre 18 y 64 años.

Para evaluar la práctica de actividad física realizada durante un período determinado de tiempo existen diferentes metodologías. Una de las más frecuentes es la utilización de cuestionarios. Este instrumento, de medición indirecta, utiliza las respuestas del sujeto para realizar una estimación de la actividad física que realiza. Las principales ventajas de los cuestionarios son que es un método no invasivo que se pueden utilizar en muestras de población grandes y representativas. Por otro lado, sus principales desventajas son que dependen mayoritariamente de la memoria y que su precisión se basa en la auto-respuesta que da el sujeto. Existen diferentes cuestionarios validados en castellano para estimar la actividad física realizada, tanto en poblaciones concretas (por ejemplo, adolescentes $^{5}$ o personas mayores ${ }^{6}$ ) como en población adulta en general ${ }^{7}$. Uno de los cuestionarios más utilizados desde hace algunos años es el International Physical Activity Questionnaire (IPAQ) ${ }^{8}$, que ha sido validado en varios idiomas, dando lugar posteriormente al Global Physical Activity Questionnaire (GPAQ) ${ }^{9}$, que proporciona información sobre la intensidad, frecuencia y duración de las actividades realizadas durante una semana. No obstante, lo relevante de un cuestionario es que permita situar a la población estudiada dentro de unos valores de referencia o en relación con las recomendaciones de práctica de actividad física. En este sentido, el GPAQ es una buena opción, de hecho existen estudios muy recientes ${ }^{10,11}$ que utilizan este instrumento, en su versión original o versión revisada. En este número se publica un interesante trabajo ${ }^{12}$ que utiliza el GPAQ y que concluye que el $20 \%$ de las personas (15-74 años) de la Comunidad de Madrid no cumplen las recomendaciones mínimas de práctica de actividad física, siendo este porcentaje del $69 \%$ si la actividad física se refiere al tiempo de ocio exclusivamente. También es necesario reseñar que existen otro tipo de cuestionarios que valoran constructos relacionados con la actividad física, como la intención de ser físicamente activo ${ }^{13}$, la fuerza del hábito $^{14} \mathrm{o}$ la autoeficacia ${ }^{15}$. Los cuestionarios que valoran estos dos últimos constructos han sido validados en castellano en el presente número de Revista Española de Salud Pública ${ }^{14,15}$.

Del mismo modo, es necesario reseñar que existen otros métodos para valorar de manera más precisa y objetiva la actividad física realizada, como son los podómetros o los acelerómetros. Los primeros son pequeños dispositivos que valoran las aceleraciones verticales. Su principal ventaja es que son discretos y fáciles de utilizar, mientras que su principal desventaja es que no valoran la intensidad de la actividad ${ }^{16}$. Por su parte, los acelerómetros valoran la actividad física realizada en uno o varios ejes. Su principal ventaja es que permiten valoraciones precisas a lo largo de varios días, analizando las diferentes intensidades utilizadas, mientras que su principal desventaja es su coste y la estandarización de su utilización ${ }^{17}$. Sin embargo, el número de estudios realizados con esta metodología ha aumentado de forma muy importante en los últimos años, en especial en niños ${ }^{18}$.

Estrechamente vinculado al ámbito de la actividad física y la Salud Pública, está el ejercicio físico, que se define como "la actividad física planificada, estructurada y repetida, cuyo objetivo es adquirir, mantener o mejorar la condición física". Así, un programa de ejercicio físico requiere la planificación y estructuración de la intensidad, volumen y tipo de activi- 
dad física que se desarrolla ${ }^{19}$. Por otro lado, la condición física relacionada con la salud fue definida en el Modelo de Toronto de Condición Física, Actividad Física y Salud ${ }^{20}$ como "un estado dinámico de energía y vitalidad que permite a las personas llevar a cabo las tareas habituales de la vida diaria, disfrutar del tiempo de ocio activo y afrontar las posibles emergencias imprevistas sin una fatiga excesiva, a la vez que ayuda a evitar enfermedades hipocinéticas y a desarrollar el máximo de capacidad intelectual experimentando plenamente la alegría de vivir". La práctica de actividad física influye en la mejora de la condición física relacionada con la salud, y en mayor medida lo hace el ejercicio físico. No obstante, algunos trabajos reflejan la relevancia de tener niveles óptimos de condición física relacionada con la salud frente a la práctica regular de actividad física de forma aislada ${ }^{21,22}$. A este respecto, la práctica de programas de ejercicio físico parece ser la forma óptima de mejorar la condición física relacionada con la salud. Sin embargo, en algunas ocasiones los programas que llegan a la ciudadanía están lejos de contar con la planificación y estructuración requerida, o no se sustentan en un soporte científico claro. Por ejemplo, son escasos los estudios sobre programas de ejercicio físico tan de moda como el aquaerobic ${ }^{23} \mathrm{o}$ el pilates ${ }^{24}$. Así, parece necesario que desde el ámbito de la Salud Pública se fomente la práctica de programas de ejercicio físico bien planificados que permitan una mejora de la condición física relacionada con la salud.

\section{BIBLIOGRAFÍA}

1. Lobstein T, Baur L, Uauy R. Obesity in children and young people: a crisis in public health. Obes Rev. 2004; 5 (suppl. 1):4-104.

2. Ortiz Marrón H, Vaamonde Martín RJ, Zorrilla Torrás B, Arrieta Blanco F, Casado López M, Medrano Albero, MJ. Prevalencia, grado de control y tratamiento de la hipertensión arterial en la población de 30 a 74 años de la Comunidad de Madrid. Estudio PREDIMERC. Rev Esp Salud Pública. 2011; 85(4): 329-338.
3. Organización Mundial de la Salud. Recomendaciones mundiales sobre actividad física para la salud. Ginebra (Suiza): Organización Mundial de la Salud; 2010 .

4. Caspersen CJ, Powell KE, Christenson GM. Physical activity, exercise and physical fitness: definitions and distinctions for health related research. Public Health Rep. 1985; 100(2): 126-131.

5. Martínez-Gómez D, Martínez-de-Haro V, Pozo T, Welk GJ, Villara A, Calle ME, Marcos A, Veiga OL. Fiabilidad y validez del cuestionario de actividad física PAQ-A en adolescentes españoles. Rev Esp Salud Pública. $2009 ; 83(3)$ : 427-439.

6. Craig CL, Marshall AL, Sjostrom M, Bauman AE, Booth ML, Pratt M, Ekelund U, Yngve A, Sallis JF, Oja P. International physical activity questionnaire: 12country reliability and validity. Med Sci Sports Exerc. 2003; 35(8): 1381-1395

7. Tuero C, De Paz JA, Márquez S. Relationship of measures of leisure time physical activity to physical fitness indicators in Spanish adults. J Sports Med Phys Fitness. 2001; 41(1): 62-67.

8. International Physical Activity Questionnaire. IPAQ [Consultado el 6 de junio de 2007] Disponible en: http://www.ipaq.ki.se

9. Armstrong T, Bull F. Development of the World Health Organization Global Physical Activity Questionnaire. J Public Health. 2006; 14: 66-70.

10. Esteghamati A, Khalilzadeh O, Rashidi A, Kamgar M, Meysamie A, Abbasi M. Physical activity in Iran: results of the third national surveillance of risk factors of non-communicable diseases (SuRFNCD-2007). J Phys Act Health. 2011; 8(1): 27-35.

11. Guthold R, Louazani SA, Riley LM, Cowan MJ, Bovet P, Damasceno A, Sambo BH, Tesfaye F, Armstrong TP. Physical Activity in 22 African Countries Results from the World Health Organization STEPwise Approach to Chronic Disease Risk Factor Surveillance. Am J Prev Med. 2011; 41(1): 52-60.

12. Rodríguez-Romo G, Cordente CA, Mayorga JI, Garrido-Muñoz M, Macias R, Lucía A, Ruiz JR. Influencia de determinantes socio-demográficos en la adherencia a las recomendaciones de actividad física en personas de entre 15 y 74 años de Madrid. Rev Esp Salud Pública. 2011; 85(4): 351-362.

13. Moreno JA, Moreno R. Cervelló EM. El autoconcepto físico como predictor de la intención de ser físicamente activo. Psicol Salud. 2007; 17(2): 261-267. 
14. Gutiérrez-Sánchez A, Pino-Justo M. Validación de la versión en español de las propiedades psicométricas de la escala SRHI (self-report habit index) para medir hábitos de ejercicio físico. Rev Esp Salud Pública. 2011; 85(4): 363-372.

15. Fernández Cabrera T, Medina Anzabo S, Herrera Sánchez IM, Rueda Méndez S, Fernández del Olmo A. Construcción y validación de una escala de autoeficiencia para la actividad física. Rev Esp Salud Pública. 2011; 85(4): 405-412.

16. Hernández LA, Ferrando JA, Quilez J, Aragonés M, Terreros JL. Análisis de la actividad física en escolares del medio urbano. Investigación en Ciencias del Deporte. 55. Madrid: Consejo Superior de Deportes, 2010.

17. Rowlands AV, Eston RG. The measurement and interpretation of children's physical activity. J Sports Sci Med. 2007; 6:270-276.

18. Rowlands AV. Accelerometer assessment of physical activity in children: An update. Pediatr Exerc Sci. 2007; 19: 252-266.

19. Rodríguez FA. Ensayos clínicos en ejercicio físico y deporte. En: X. Bonfill (Ed.). Ensayos clínicos en intervenciones no farmacológicas (pp.23-35). Barcelona: Fundación Dr. Antonio Esteve, 2001.

20. Bouchard C, Shepard RJ, Stephens T. Physical activity, fitness, and health. Champaign: Human Kinetics, 1994.

21. Erikssen G. Physical fitness and changes in mortality: the survival of the fittest. Sports Med. 2001; 31:571-576.

22. Myers J, Kaykha A, George S, Abella J, Zaheer N, Lear S, Yamazaki T, Froelicher V. Fitness versus physical activity patterns in predicting mortality in men. Am $\mathrm{J}$ Med. 2004; 117: 912-918.

23. Saavedra JM, De la Cruz E, Escalante Y., Rodríguez FA. Influence of a medium-impact aquaerobic program on health-related quality of life and fitness level in healthy adult females. J Sports Med Phys Fitness. 2001; 47(4): 468-474.

24. Cruz-Ferreira A, Fernandes J, Gomes D, Bernardo LM, Kirkcaldy BD, Barbosa TM, Silva A. Effects of pilates-based exercise on life satisfaction, physical selfconcept and health status in adult women. Women Health. 2011; (3): 240-255. 